\title{
The correlation between pre-operative ultrasonographic median nerve evaluation and the operative procedure in CTS
}

\author{
Ahmad Fouad Abdelbaki Allam (1D, Ahmed Fathy Sadek', Manal Fayez AbuSamra², Ahmed Hamed Ismail ${ }^{3}$ and \\ Mohammad Fouad Abdel Baki Allam²*
}

\begin{abstract}
Background: Ultrasonography is a convenient non-invasive diagnostic tool with wide availability and cost effectiveness. Musculoskeletal ultrasonography is a growing field used for diagnosis of many musculoskeletal disorders; carpal tunnel syndrome (CTS) could be one of these disorders. The purpose of this study was to assess the impact of median nerve ultrasonography in carpal tunnel syndrome on surgical management, and its added value in operative techniques selection, and in tailoring the surgical steps. Thirty patients with CTS and thirty normal subjects underwent superficial ultrasonography. The cross-sectional area at different levels and flattening ratios were measured on both groups. The patients who had failed conservative treatment had surgical carpal tunnel release.
\end{abstract}

Results: There was significant positive correlation between the need for external neurolysis with increased flattening ratio with cutoff values $>4$, and between the need for approach extension with increased cross-sectional area difference between pisiform level and pronator quadratus level with cutoff values of cross-sectional area difference $>7$. Conclusions: Ultrasonographic measurements of median nerve in CTS could be helpful in selection and modification of operative procedure including the surgical approach and need for neurolysis.

Keywords: Median nerve, Ultrasonography, Carpal tunnel syndrome, Carpal tunnel release

\section{Background}

Carpal tunnel syndrome (CTS) is the most frequently encountered upper limb compression neuropathy; it mostly occurs in patients subjected to repetitive wrist motion [1].

Median nerve compression in CTS results in neural enlargement which could be expressed radiologically by the increase in cross-sectional area (CSA) $[2,3]$.

It was found that median nerve CSA enlargement is a specific criterion for the radiological diagnosis of CTS [4-6].

Different imaging tools have been utilized for assessment of MN in CTS; they vary from the simple cost-effective tool as ultrasonography to more complex one as MRI, which

\footnotetext{
*Correspondence: twins_drs@hotmail.com; afallam@mu.edu.eg

${ }^{2}$ Department of Radiology, Faculty of Medicine, Minia University, Minya

61512, Egypt
}

Full list of author information is available at the end of the article could include quantitative methods, diffusion-weighted imaging, and diffusion tensor imaging [7-9].

Ultrasonography is a non-invasive, widely available and cost effective imaging tool could be used in diagnosis of CTS. Normally, the median nerve CSA does not vary greatly along its course. Ultrasonography could elucidate the enlargement in the median nerve which occurs at or just proximal to the carpal tunnel level. There is no consensus on which value of median nerve CSA used for CTS diagnosis; most studies found a cutoff value ranging from 9 to $12 \mathrm{~mm}^{2}$ [10-14].

Chronic patients with intermittent, advanced, persistent, or progressive syndromes and who have failed conservative treatment are better treated with carpal tunnel release [15]. 


\section{Aim of the work}

The purpose of this study was to assess the impact of median nerve ultrasonography in carpal tunnel syndrome (CTS) on surgical management, and its added value in operative techniques selection, and in tailoring the surgical steps.

\section{Methods}

\section{Study participants}

Between March 2017 and March 2018, after ethics committee approval, thirty patients with CTS and other thirty asymptomatic volunteers were included in the study.

\section{Inclusion criteria}

The CTS diagnosis was primarily based on the following:

1. Positive Phalen's test (less than $30 \mathrm{~s}$ )

2. Positive Tinel's sign

3. Absence of cervical radiculopathy along $\mathrm{C} 6$ and $\mathrm{C} 7$ roots

4. The diagnosis was confirmed by electrophysiological nerve conduction studies that were considered abnormal when:
a. Distal motor latency of $>4.5 \mathrm{~ms}$
b. And/or sensory latency of $>3.5 \mathrm{~ms}$
c. And/or median-ulnar nerve sensory latency difference in peak latencies of $>0.4 \mathrm{~ms}$

Exclusion criteria: bifid median nerve and carpal tunnel masses as ganglion cysts and neurofibroma

The thirty asymptomatic volunteers were recruited in the study as a control group, their inclusion criteria were (a) adult with age group from third to fifth decades, (b) no history of hand pain/numbness, (c) negative Phalen's test and negative Tinel's sign at either wrist.

A written informed consent has been obtained from all study participants.

\section{Study technique}

All the participants were subjected to superficial ultrasonography using the Toshiba ultrasonography machine (Xario 200) with a multi-frequency linear array transducer (selected frequency $12 \mathrm{MHz}$ ).

The volunteers were examined on both wrists, whereas the patients were examined on the symptomatic wrists.

The patients were examined while sitting, with a supinated hand. The transducer was kept perpendicular to the $\mathrm{MN}$ at all levels, reducing the anisotropy effect and improving the visualization of MN borders. All measurements were assessed in axial plane.

The ultrasonographic protocol examined the $\mathrm{MN}$ dimensions at easily obtained three axial levels, all outcome data was quantitative; the protocol excluded any subjective median nerve features such as echogenicity changes, restricted motion during finger flexion and extension, and longitudinal notch signs.

The cross-sectional area (CSA) of the median nerve was measured in transverse plane at two levels:

1. At the pisiform bone (PS) within the proximal carpal tunnel (Fig. 1).

2. Over the transversely oriented fibers of the pronator quadratus muscle (PQ) before entering the carpal tunnel (Fig. 1).

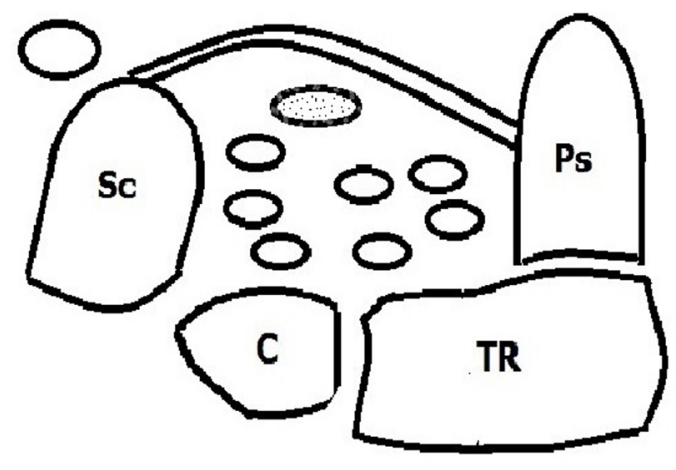

A

Fig. 1 a lllustration drawing for CSA calculation of the median nerve. $\mathbf{b}$ Ultrasonographic cross-sectional area calculation of the median nerve at the level of pisiform bone (area $A=18 \mathrm{~mm} 2$ ) and at the level of pronator quadratus muscle (area $B=11 \mathrm{~mm} 2) ; C S A$ difference $=(18-11)=7$. SC, scaphoid; C, capitate; TR, triquetrum; PS, pisiform 
The study used a freehand area tool on the ultrasound machine for CSA measurement to establish high accuracy of the measurements. The CSA difference between the two levels (PS-PQ) was calculated.

The flattening ratio (FR) at the distal carpal tunnel opposite the hamate was measured also in the axial plane by dividing the median nerve transverse diameter by the anteroposterior diameter (Fig. 2).

\section{Treatment and operative details}

All patients received conservative treatment in addition to wrist splinting for 4-6 weeks.

Surgical management was considered in 24 patients after failed conservative treatment with persistent signs and symptoms. After written informed consents, surgery for carpal tunnel release was performed as 1-day surgery under general anesthesia, using open (mini-palm) longitudinal approach.

Incision at the intersection of the Kaplan cardinal line and the radial border of the fourth ray ending at the wrist crease begins distally at the Kaplan cardinal line and extends proximally approximately $2 \mathrm{~cm}$, dissection through the subcutaneous fat, and palmaris brevis muscle to expose the transverse carpal ligament, release the most ulnar aspect of the ligament close to the hook of hamate under direct visualization, release the distal forearm fascia proximally, explore the median nerve to ensure adequate decompression versus the possible need for either wound extension or external neurolysis, superficial skin closure using 3-0 nylon, place the hand in a soft compressive dressing.

\section{Statistical analysis}

Results were recorded and represented as range, means \pm standard deviations (SD). Statistical analysis was done using SPSS, 16 (SPSS Inc, IL, USA). Independent $t$ test and Mann-Whitney test were used for normal and nonnormal quantitative data respectively. Correlation coefficient $(r)$ was calculated using Spearman correlation test to find out the linear relationship between ultrasonographic and operative data.

$P$ values less than 0.05 were considered statistically significant.

\section{Results}

The study group included twelve males and eighteen females with mean age $43.93 \pm 4.51$ (range $35-52$ years), whereas the control group included fifteen males and fifteen females, with their mean age $36.7 \pm 4.86$ (range 29-45 years).

\section{Descriptive statistics}

The descriptive statistics of median nerve dimensions of the study and control groups was summarized in Table 1. Comparison between the two groups showed higher values in CTS group regarding CSA at the pisiform, CSA difference (PS-PQ), and FR at the hamate; these differences were statistically significant.

Twenty four out of the thirty patients had failed conservative treatment and had received operative carpal tunnel release using (mini-palm) open longitudinal approach. They were six males and eighteen females with mean age $44.13 \pm 4.19$ (range 37-52 years). The surgical team was blinded to the ultrasonographic findings of the median nerve.

The other six patients showed satisfactory results with conservative treatment, all were males, their mean age was $42.5 \pm 5.01$ (range $35-48$ years).

All the operatively treated patients were followed up for 6 months with $0 \%$ infection rate, complete resolution

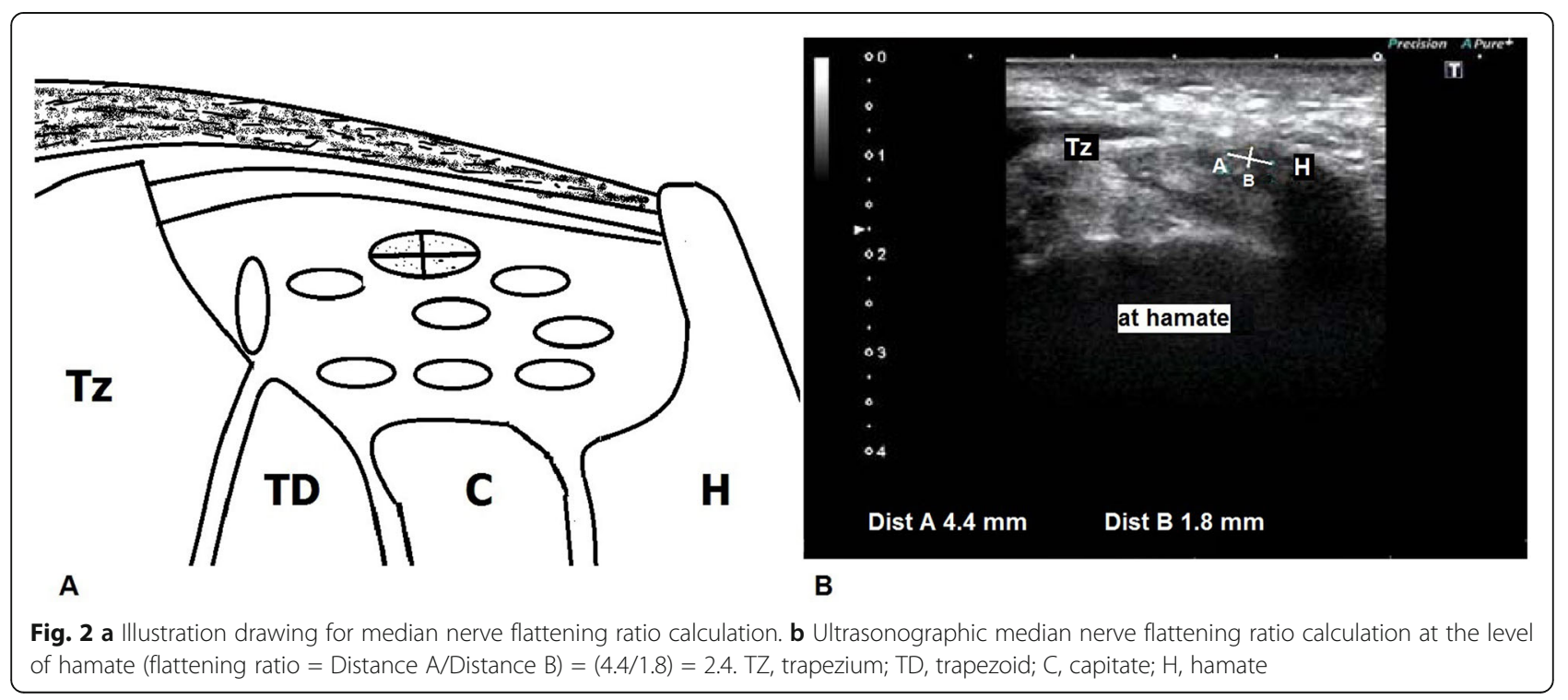


Table 1 Comparison between MN dimensions in normal individuals and CTS patients

\begin{tabular}{llll}
\hline MN variable & Normal $(n=60$ wrists $)$ & CTS $(n=30)$ & $P$ \\
\hline $\begin{array}{llll}{ }^{(1)} \text { Area PQ } \\
\text { Range }\end{array}$ & $(8-10)$ & $(8-11)$ & 0.305 \\
Mean \pm SD & $9.28 \pm 0.58$ & $9.46 \pm 1.11$ & \\
${ }^{(1)}$ Area PS & & $(10-20)$ & $<\mathbf{0 . 0 0 1}$ \\
Range & $(9,10)$ & $14.26 \pm 2.91$ & \\
Mean \pm SD & $9.86 \pm 0.34$ & & $<\mathbf{0 . 0 0 1}$ \\
${ }^{(1)}$ Flattening ratio & & $(2.2-4.3)$ & \\
Range & $(2.2-2.8)$ & $3.68 \pm 0.71$ & \\
Mean \pm SD & $2.46 \pm 0.16$ & & \\
${ }^{(2)}$ Area PS- Area PQ & & $(1-10)$ & \\
Range & $(0-1)$ & $4.76 \pm 2.34$ & \\
Mean \pm SD & $0.58 \pm 0.49$ & & \\
\hline
\end{tabular}

CSA cross-sectional area, $P Q$ pronator quadratus muscle, $P S$ pisiform bone ${ }^{(1)}$ Independent sample $t$ test for parametric quantitative data

${ }^{(2)}$ Mann-Whitney test for non-parametric quantitative data

Significant difference at $p$ value $<0.05$

of symptoms had observed at a mean duration $3.8 \pm 5.2$ week (range 3-8 weeks).

At 6 month follow up, there were no recorded cases of recurrence or persistence of symptoms.

The MN dimensions of non-surgically treated patients were lower than that of the surgically treated patients and the differences were statistically insignificant. There was a significant difference between the median nerve CSA at the pisiform and at the pronator quadratus in all patients (Table 2).

Table 2 Comparison between the MN dimensions of the surgically and non-surgically treated patients

\begin{tabular}{|c|c|c|c|}
\hline MN variable & $\begin{array}{l}\text { Surgical patients } \\
(n=24)\end{array}$ & $\begin{array}{l}\text { Non-surgical } \\
\text { patients }(n=6)\end{array}$ & $P$ \\
\hline$\overline{(1)}$ Area PQ & $P<0.001$ & $P<0.001$ & \\
\hline Range & $(8-11)$ & $(8-11)$ & \\
\hline Mean \pm SD & $9.42 \pm 1.1$ & $9.67 \pm 1.2$ & 0.64 \\
\hline \multicolumn{4}{|l|}{${ }^{(1)}$ Area PS } \\
\hline Range & $(10-20)$ & $(11-14)$ & \\
\hline Mean \pm SD & $14.25 \pm 3.01$ & $12.67 \pm 1.5$ & 0.32 \\
\hline \multicolumn{4}{|c|}{ (1)Flattening ratio } \\
\hline Range & $(2.2-4.3)$ & $(2.2-4)$ & \\
\hline Mean \pm SD & $3.68 \pm 0.71$ & $3.13 \pm 0.74$ & 0.07 \\
\hline \multicolumn{4}{|c|}{${ }^{(2)}$ Area PS-Area PQ } \\
\hline Range & $(1-10)$ & $(2-4)$ & \\
\hline Mean \pm SD & $4.83 \pm 2.44$ & $3 \pm 0.89$ & 0.106 \\
\hline
\end{tabular}

CSA cross sectional area, $P Q$ pronator quadratus muscle, $P S$ pisiform bone (1) Independent sample $t$ test for parametric quantitative data

${ }^{(2)}$ Mann-Whitney test for non-parametric quantitative data

significant difference at $p$ value $<0.05$

\section{Correlation analysis}

Intraoperative external neurolysis of the median nerve was found to be necessary in twelve patients to free the nerve trunk from surrounding fibrous tissue that was seen adherent to the superficial epineurium, and all these patients had flattening ratio $>4$ (Fig. 3).

There was a significant positive correlation between the need for external neurolysis and the flattening ratio, the correlation coefficient was high $(r=0.69, P$ $<0.0001$ ) (Table 3).

There was an insignificant positive correlation between the need for external neurolysis and CSA difference (PS$\mathrm{PQ})$, the correlation coefficient was low $(r=0.21, P=0.16)$ (Table 3).

Intraoperative severe median nerve compression was observed in five patients as marked volar bulging of the median nerve after incision of the transverse carpal ligament with bulbous nerve swelling required-in the surgeon preference-extending the (mini-palm) longitudinal incision proximal to the flexor crease of the wrist for adequate and under vision division of the distal forearm deep fascia, all these five patients had CSA difference (PS-PQ) $>7$ (Fig. 4).

Correlating this need for approach extension with the CSA difference (PS-PQ) resulted in a significant positive correlation with high correlation coefficient $(r=0.68, P$ $=0.0001)$ (Table 3).

There was a significant positive reasonable correlation $(r=0.42, P=0.0205)$ when correlating the need for approach extension with the flattening ratio, these five patients had flattening ratio $>4.2$ despite there were another three patients with flattening ratio $>4.2$ had no need for approach extension (Table 3).

Correlation between the flattening ratio of the surgical patients and the electrophysiological nerve conduction studies resulted in significant positive correlation between the flattening ratio and motor distal latency with high correlation coefficient $(r=0.63, P=0.0005)$ and significant negative correlation between the flattening ratio and motor amplitude $(r=-0.69, P<0.0001)$ (Table 4$)$.

The mean post-operative follow-up period was $5.6+$ 1.2 months. Twenty-one patients $(87.5 \%)$ were symptomfree, and twenty two patients $(91.6 \%)$ had normal function. There was an insignificant difference between the patients who had extended approach and/or external neurolysis and the others.

\section{Discussion}

The entrapment of the MN occurs between the transverse carpal ligament and carpal bones, with subsequent CSA enlargement which occurs at the level of proximal carpal tunnel (the level of pisiform), the MN size appears to be not affected at more proximal level above the carpal tunnel (the level of pronator quadratus) [12]. 


\section{Flattening ratio}

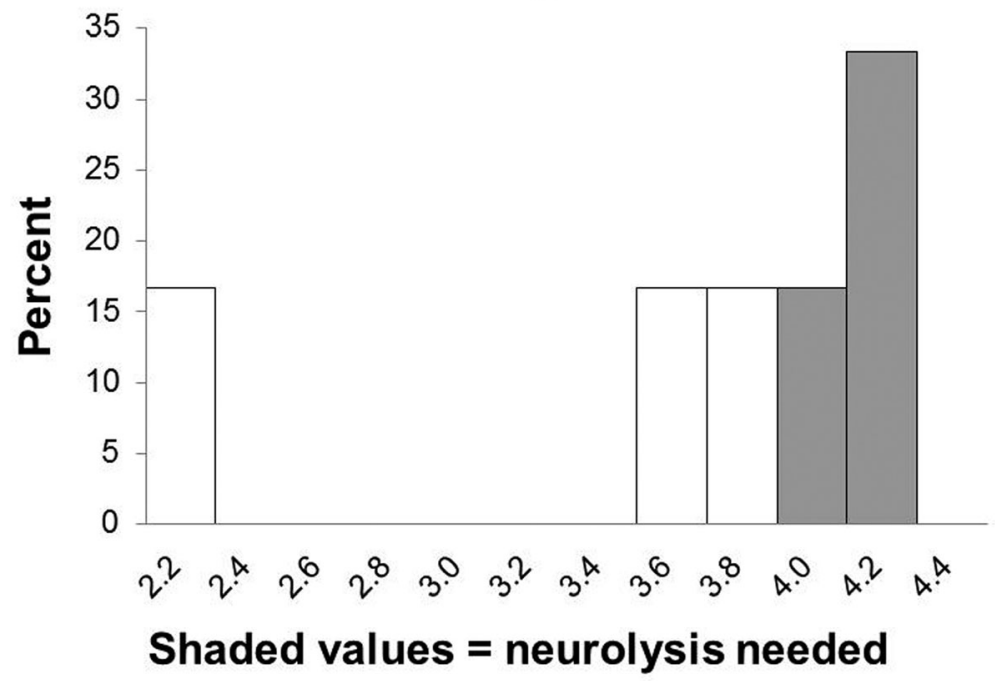

Fig. 3 Histogram of the flattening ratio and need for neurolysis

Many sonographic studies had compared MN dimensions between CTS patients and controls and found that CSA at the proximal carpal tunnel was higher in CTS patients than control group. Accordingly, the CSA difference (PS-PQ) was also higher in CTS patients than the control group [16-19].

Sonographic median nerve FR was also assessed as a differentiating parameter for neuropathy and a cutoff value of 3 was accepted to be significant for CTS $[16,17,20]$.

The current study showed that CTS patients had statistically significant higher values of CSA at the pisiform, CSA difference (PS-PQ) and FR at the hamate.

In the current study, the CSA at the pisiform, CSA difference (PS-PQ), and FR in the non-surgically treated patients were lower than that of the surgically treated patients, these differences were statistically insignificant and this might be due to small population size.

Surgical approaches for carpal tunnel release include longitudinal approach mini-palm or extended open, double incision, transverse mini incision, and endoscopic carpal tunnel release. The longitudinal approaches

Table 3 Correlation between the relevant operative data and ultrasonographic data

\begin{tabular}{llllll}
\hline Correlation variables & \multicolumn{2}{l}{ Flattening ratio } & & \multicolumn{2}{l}{ PS-PQ CSA } \\
\cline { 2 - 2 } & $r$ & & $P$ & & $P$ \\
\hline Need for neurolysis & 0.69 & $<0.0001$ & & 0.21 & 0.16 \\
Need for approach extension & 0.42 & 0.0205 & & 0.68 & 0.0001 \\
\hline $\begin{array}{l}\text { Spearman Correlation P probability, } r \text { correlation coefficient } \\
r=0-0.2, \text { very low and probably meaningless; } r=0.2-0.4 \text {, a low correlation }\end{array}$ \\
$r=0.4-0.6$, a reasonable correlation; $r=0.6-0.8$, a high correlation \\
$r=0.8-1.0$, a very high correlation; $P<0.05=$ significant
\end{tabular}

permit the surgeon to extend the incision when there is an indication for more release [21-23].

To date, there is no publication assesses the impact of preoperative median nerve ultrasonography on surgical decisions.

In the current study, the data of median nerve ultrasonography was analyzed retrospectively on the light of the operative notes, and it was found that the mini-palm longitudinal approach had to be extended in five patients for adequate decompression; and all these five patients had median nerve CSA difference (PS-PQ) > 7 with significant-high correlations.

This CSA difference could be recommended as a cutoff value at which surgeon should put in mind that surgical field extension might be demanded.

External neurolysis means the removal of fibrotic tissue outside a nerve trunk. Median nerve neurolysis is not a routine step in primary carpal tunnel release; however, external neurolysis could be needed if fibrous adhesions are found intra operatively [24].

Asami A et al. studied external neurolysis of the median nerve in severe CTS cases who had no electrophysiologic reaction; they found that all patients regained some degree of hand function post-operatively, so they recommended external neurolysis for severe CTS to achieve satisfactory results [24].

There is no consensus on specific preoperative advisory measures to recommend planning external neurolysis.

In the current study, twelve patients needed intraoperative external neurolysis of the median nerve to free the nerve trunk from surrounding fibrous tissue that was seen adherent to the superficial epineurium; all of them had median nerve flattening ratio $>4$ with significant- 


\section{PS-PS CTS}



Fig. 4 Histogram of the cross sectional area difference and need for wound extension

high correlations. This value could be assigned as a cutoff value at which surgeon should put the possible need of median nerve external neurolysis in mind.

The high FR may reflect severe nerve compression, and long-standing severe compression may lead to fibrosis around the nerve.

The flattening ratio was correlated significantly with both motor distal latency and motor amplitude, this could reflect the reliability of the flattening ratio as a parameter of the severity of carpal tunnel syndrome.

The need for either neurolysis or wound extension might consider choosing a traditional open longitudinal approach rather than endoscopic or "minimal-incision" as transverse approach to permit possible approach extension.

The current study could define cutoff values of flattening ratio $>4$ for the possible need for external neurolysis and cutoff values of CSA difference (PS-PQ) $>7$ for possible need for wound extension.
Five patients out of eight with flattening ratio $>4.2$ were found to need intraoperative wound extension for adequate decompression. Although the correlation was significant and reasonable, this might require a larger sample size study for more accurate evaluation.

To the best of the authors' knowledge, no study up to date was conducted to evaluate the implication of the ultrasonographic data of median nerve dimensions on surgical carpal tunnel release.

\section{Conclusions}

Ultrasonography of median nerve in CTS could assist selection of operative approach and decision, recommending choosing longitudinal approach when flattening ratio $>4$ and/or cross-sectional area difference between pisiform level and pronator quadratus level $>7$ to allow for the possible need for wound extension

Median nerve external neurolysis could be recommended when flattening ratio of median nerve $>4$.

Table 4 Correlation between the flattening ratio and electro-diagnostic study parameters

\begin{tabular}{|c|c|c|c|}
\hline & \multirow[t]{2}{*}{ Factors (range) mean \pm SD } & \multicolumn{2}{|c|}{$N=24$} \\
\hline & & $r$ & $p$ \\
\hline \multirow[t]{6}{*}{ Flattening ratio } & Motor distal latency (ms) (3.6-8) $5.021 \pm 1.351$ & 0.63 & 0.0005 \\
\hline & Motor amplitude $(\boldsymbol{m v})(2-7) 4.346 \pm 1.526$ & -0.69 & $<0.0001$ \\
\hline & Motor nerve conduction velocity $(\mathrm{m} / \mathrm{s})(15-55) 38.33 \pm 12.13$ & -0.59 & 0.0013 \\
\hline & Sensory peak latency (ms) (3.3-6) $4.258 \pm 0.756$ & 0.36 & 0.042 \\
\hline & Sensory amplitude (mv) (10-18) $14.42 \pm 2.32$ & -0.24 & 0.1314 \\
\hline & Sensory nerve conduction velocity(m/s) (15-40) $26.46 \pm 7.29$ & -0.26 & 0.1099 \\
\hline
\end{tabular}


The need for wound extension should be put in mind when the median nerve cross-sectional area difference between pisiform level and pronator quadratus level $>7$.

Larger sample size study is needed for more validation of linear relationship between the surgical details and sonographic cutoff values.

\section{Abbreviations}

CST: Carpal tunnel syndrome; CSA: Cross-sectional area; FR: Flattening ratio; MN: Median nerve; PQ: Pronator quadratus muscle; PS: Pisiform

\section{Acknowledgements}

Not applicable.

\section{Authors' contributions}

MFAA, MFAS and AHI carried out the ultrasonographic technique and participated in the study analysis. AHI carried out the electrophysiological nerve conduction studies AFAA and AFS carried out the clinical assessment and the operative techniques of the cases and participated in the design of the study AFAA performed the statistical analysis. The author(s) read and approved the final manuscript.

\section{Funding}

This study had no funding from any resource.

\section{Availability of data and materials}

The datasets used and/or analyzed during the current study are available from the corresponding author on reasonable request.

\section{Ethics approval and consent to participate}

This study was approved by the Research Ethics Committee of the Faculty of Medicine at Minia University in Egypt on March 2017 (Reference number is not applicable). All subjects included in this study signed written informed consent to participate in this research.

\section{Consent for publication}

All subjects included in this research gave written informed consent to publish the data contained within this study.

\section{Competing interests}

The authors declare that they have no competing interests.

\section{Author details}

'Department of Orthopedic Surgery, Faculty of Medicine, Minia University, Minya, Egypt. ${ }^{2}$ Department of Radiology, Faculty of Medicine, Minia University, Minya 61512, Egypt. ${ }^{3}$ Department of Rheumatology, Faculty of Medicine, Minia University, Minya, Egypt.

Received: 1 June 2020 Accepted: 26 June 2020

Published online: 08 July 2020

\section{References}

1. Lo JK, Finestone HM, Gilbert K, Woodbury MG (2002) Community based referrals for electrodiagnostic studies in patients with possible carpal tunnel syndrome what is the diagnosis? Arch Phys Med Rehabil 83:598-603

2. Gelberman RH, Eaton R, Urbaniak JR (1994) Peripheral nerve compression. Instr Course Lect 43:31-53

3. Rojviroj S, Sirichativapee W, Kowsuwon W, Wongwiwattananon J et al (1990) Pressures in the carpal tunnel. A comparison between patients with carpal tunnel syndrome and normal subjects. J Bone Joint Surg (Br) 72:516-518

4. Buchberger W (1997) Radiologic imaging of the carpal tunnel. Eur J Radiol 25:112-117

5. Sarria L, Cabada T, Cozcolluela R, Berganza TM, Garcia S (2000) Carpal tunnel syndrome: usefulness of sonography. Eur Radiol 10:1920-1925

6. Wong SM, Griffith JF, Hui ACF, Tang A, Wong KS (2002) Discriminatory sonographic criteria for the diagnosis of carpal tunnel syndrome. Arthritis Rheum 46:1914-1921
7. Allam M, Ibrahiem M, Allam A (2017) The value of quantitative MRI using 1. 5 T magnet in diagnosis of carpal tunnel syndrome. EJRNM 48:201-206. https://doi.org/10.1016/j.ejrnm.2016.11.008

8. Chianca V, Albano D, Messina C, Cinnante CM, Triulzi FM, Sardanelli F, Sconfienza LM (2017) Diffusion tensor imaging in the musculoskeletal and peripheral nerve systems: from experimental to clinical applications. Eur Radiol Exp 1(1):12. https://doi.org/10.1186/s41747-017-0018-1

9. Vetrano IG, Sconfienza LM, Albano D, Chianca V, Nazzi V. Recurrence of carpal tunnel syndrome in isolated non-syndromic macrodactyly: DTI examination of a giant median nerve. Skeletal Radiol. 2018; Oct 20. doi: https://doi.org/10.1007/s00256-018-3098-y.

10. Beekman R, Visser LH (2003) Sonography in the diagnosis of carpal tunnel syndrome: a critical review of the literature. Muscle Nerve 27:26-33

11. Budisic M, Bosnjak J, Lovrencic-Huzjan A, Strineka M, Bene R, Azman D (2008) Transcranial sonography in the evaluation of pineal lesions: two-year follow up study. Acta Clin Croat 47:205-210

12. Heinemeyer O, Reimers CD (1999) Ultrasound of radial, ulnar, median, and sciatic nerves in healthy subjects and patients with hereditary motor and sensory neuropathies. Ultrasound Med Biol 25:481-485

13. Koenig R, Pedro M, Heinen C, Schmidt T (2009) Highresolution ultrasonography in evaluating peri-pheral nerve entrapmentand trauma. Neurosurg Focus 26:E13

14. Yucel A, Yilmaz O, Babaoglu S, Acar M, Degirmenci B (2008) Sonographic findings of the median nerve and prevalence of carpal tunnel syndrome in patients with Parkinson's disease. Eur Radio1 67:546-550

15. English JH, Gwynne-Jones DP (2015) Incidence of carpal tunnel syndrome requiring surgical decompression: a 10.5-year review of 2,309 patients. J Hand Surg $[\mathrm{Am}]$ 40:24-27

16. Buchberger W, Judmaier W, Birbamer G, Lener M, Schmidauer C (1992) Carpal tunnel syndrome: diagnosis with high resolution sonography. AJR Am J Roentgenol 159:793-798

17. Duncan I, Sullivan P, Lomas F (1999) Sonography in the diagnosis of carpal tunnel syndrome. AJR Am J Roentgenol 173:681-684

18. Klauser AS, Halpern EJ, De ZT, Gudrun M, Feuchtner GM et al (2009) Carpal tunnel syndrome assessment with US: value of additional cross-sectional area measurements of the median nerve in patients versus healthy volunteers. Radiology 250:171-177

19. Yesildag A, Kutluhan S, Sengul N, Koyuncuoglu HR, Oyar O (2004) The role of ultra sonographic measurements of the median nerve in the diagnosis of carpal tunnel syndrome. ClinRadiol 59:910-915

20. Aiman D, Bosnjak J, Strineka M, Bene R, Budisic M (2009) Median nerve imaging using high-resolution ultrasound in healthy subjects. Acta Clin Croat 48:265-269

21. Alves MPT. Transverse mini-incision for carpal tunnel release. Acta ortop bras. [online] 2011;19:362-67.

22. Citron ND, Bendall SP (1997) Local symptoms after open carpal tunnel release. A randomized prospective trial of two incisions. J Hand Surg (Br) 22: 317-321

23. Wilson KM (1994) Double incision open technique for carpal tunnel release: an alternative to endoscopic release. J Hand Surg [Am] 19:907-912

24. Asami A, Tsuruta T, Asami T, Nishikawa H, Onoyama Y, Watanabe $H$. External neurolysis applied to severe carpal tunnel syndrome.orthopaedics and traumatology [in Japanese] 1992; 41:127-30. doi:https://doi.org/10.5035/ nishiseisai.41.127

\section{Publisher's Note}

Springer Nature remains neutral with regard to jurisdictional claims in published maps and institutional affiliations. 